\title{
HRTS ULTRAVIOLET SOLAR SPECTROSCOPY
}

\author{
K.P. Dere \\ E.O. Hulburt Center for Space Research \\ Naval Research Laboratory \\ Washington, D.C. 20375-5000, USA
}

The NRL High Resolution Telescope and Spectrograph (HRTS) consists of a telescope, stigmatic UV spectrograph, UV broadband spectroheliograph and $\mathrm{Ha}$ film and video cameras. An image of the Sun is focussed onto the slit jaws of the spectrograph by a $30 \mathrm{~cm}$ Cassegrain telescope with a spatial resolution of $1 "$. The stigmatic UV spectrograph employs a tandem-Wadsworth mount and photographically records spectra along the $1000 "$ ( 1 solar radius) slit with a resolution of $50 \mathrm{~m} \AA$ in the $1170-1710 \AA$ wavelength region. Images of the slit jaws in a tunable $100 \AA$ bandpass are produced on film by the UV spectroheliograph which uses a reversed tandem-Wadsworth mount. The slit jaws are also viewed through an $\mathrm{Ha}$ filter by video and film cameras. To date, the HRTS instrument has flown on four rocket flights and is being prepared for flight on Spacelab-2.

The data are returned as simultaneous stigmatic spectra of the complete 1170-1710 $\AA$ spectrum for each individual feature imaged on the slit. Visual inspections of the spectra turned up a number of phenomena which showed distinctive Doppler shifts. The first of these were termed "coronal jets" (Brueckneq and Bartoe ${ }_{5}$ 1983). They consist of plasma at 2 temperatures from $2 \times 10^{4} \mathrm{~K}$ to $2 \times 10^{5} \mathrm{~K}$ which are accelerated at $5 \mathrm{~km} \mathrm{~s}$ up to velocities of $400 \mathrm{~km} \mathrm{~s}^{-1}$, roughly the coronal sound speed. In one case, four of these jets developed sequentially at the same location during a four minute rocket flight. Their extent along the slit is typically $7000 \mathrm{~km}$. Erupting magnetic loops or bubbles would be consistent with the observed spectra. Brueckner and Bartoe suggested that there is enough mass, momentum and energy in these jets to cause the solar wind. Another possibility is that these are the small scale end of a range of eruptive phenomena, including coronal transients that are as a whole responsible for the production of the solar wind.

Another category of events with distinctive Doppler signatures is the so-called "explosive event." These have Doppler shifts (red, blue or both) on the order of $100 \mathrm{~km} \mathrm{~s}^{-1}$ and spatial scales which are often near the instrumental resolution. This category encompasses a wide range, not all of which are obviously related. However, in general, these features are distributed isotropically over the solar disk and there is no apparent correlation between their Doppler signature and solar latitude. Whether they are observed above the limb or on the disk, they appear to be more or less similar. From this it can be concluded that, in general, the flow velocities are isotropic as might result from an explosion. A wide range of timescales, from 20 s to greater than 200 s, is evident. There does appear to be a distinct subclass which are called "turbulent events," which are distinguished by the symmetry of their Doppler shifts that may be the result of enhanced turbulent velocities in the emitting plasma. 
The two sunspots observed on the disk during separate flights both show high-speed downflows of chromospheric and transition zone material. In both cases, the sunspot contained a light bridge, but the nature of the flow pattern were considerably different. In the first case, small regions ( $1000 \mathrm{~km}$ across) were found scattered throughout the spot. The downflow velocities were on the order of $100-150 \mathrm{~km} \mathrm{~s}$. In the second case, a continuum of downflow velocities from $0 \mathrm{~km} \mathrm{~s}^{-1}$ up to a maximum of about $175 \mathrm{~km} \mathrm{~s}^{-1}$ were found throughout the area of the spot, with maximum velocity and intensity attained over the light bridge.

In the supergranular cell centers, the lines of the strong $C I$ multiplets near $1561 \AA$ and $1657 \AA$ show smalf scale (2000 km FWHM) structures that are the site of $10-20 \mathrm{~km} \mathrm{~s}^{-1}$ flows which we refer to as chromospheric jets (Dere, Bartoe and Brueckner 1983). Both red and blue shifts are seen although the blue shifts are brighter and more numerous. Their average lifetime is on the order of $40 \mathrm{~s}$. There is considerable opacity in the C I lines so that the velocities deduced from the line profiles may be exaggerated by radiative transfer effects. The chromospheric jets are best seen in the C I lines but also appear in most chromospheric lines. In Si I, the deduced Doppler shifts are on the order of $3-5 \mathrm{~km} \mathrm{~s}^{-1}$. Since these events occur in the cell center, the C IV intensity is on average quite low. Nevertheless, there is no indication from C IV emission that these chromospheric jets ever reach transition zone temperature $\left(3 \times 10^{4} \mathrm{~K}\right.$ or greater). What is surprising is that events such as these are found onfy in the cell centers. Spicules, when seen at the limb, show a $25 \mathrm{~km} \mathrm{~s}^{-1}$ outward velocity and it is generally assumed that spicules are found on the disk in the supergranular cell boundaries. We find no evidence of $25 \mathrm{~km} \mathrm{~s}^{-1}$ spicular flows in any of our chromspheric lines in the boundaries.

More typical profiles of optically thin chromospheric and transition zone lines are Gaussian with subsonic Doppler shifts. The wavelength scale on which these Doppler shifts are measured is derived from measurements of the numerous narrow Si I lines in this wavelength region. The Si I lines themselves show small flow velocities with a standard deviation of $2 \mathrm{~km} \mathrm{~s}$. The measurement of the Doppler shift of any individual line profile has an accuracy of about $2 \mathrm{~km} \mathrm{~s}^{-1}$. Ions such as $C$ IV and $F e$ II have multiple lines in the spectrum so that the errors in their measured Doppler shifts can be reduced through averaging. Chromospheric lines of Fe II show RMS Doppler shifts of $+2.4 \mathrm{~km} \mathrm{~s}^{-1}$ about a near zero average Doppler shift. Again, there are no spicular $\left(25 \mathrm{~km} \mathrm{~s}^{-1}\right.$ ) velocities derived from the $\mathrm{Fe}$ II profiles. The C IV disk velocity distribution is characterized by an average red shift (downflow) of $5 \mathrm{~km} \mathrm{~s}^{-1}$ with a standard deviation of $6 \mathrm{~km} \mathrm{~s}^{-1}$. Clearly, there are very few blue shifts found and most that are tend to be associated with the explosive events. Syphon flows, therefore, cannot play any significant role in describing transition zone flow patterns. Pneumann and Kopp (1977) suggested that this downward enthalpy flux should be a significant source of the energy that is lost in the transition zone through radiation. If so, one might expect to find a correlation between 
local downflow velocity and intensity. However, the data shows no such correlation. It has also been suggested that the turbulent velocities derived from transition zone line profiles are the result of propagating acoustic (Boland et al., 1973) or Alfven waves which could be an

alternate source of transition zone heating. The HRTS data shows a relatively high correlation between C IV line intensity and line width for the near-Gaussian profiles, in support of these ideas (Dere, Bartoe and Brueckner, 1984).

From the data obtained on the third rocket flight, time series of profiles covering a duration of 200 s at 20 s intervals have been constructed. Aside from the chromospheric jets, chromospheric lines show small temporal variations and smooth variations along the slit. On the other hand, the time series of C IV spectra is made up of distinct

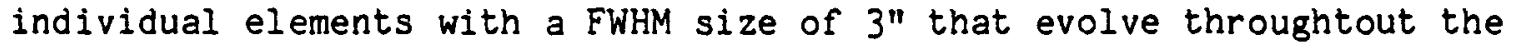
rocket flight. Three such elements have been studied in some detail. Two show only small fluctuations (10\% RMS) in intensity with small accelerations in downflow velocity of $2-5 \mathrm{~km} \mathrm{~s}^{-1}$ in 200s. A third shows a strong rise in red shift from $15 \mathrm{~km} \mathrm{~s}^{-1}$ to $22 \mathrm{~km} \mathrm{~s}^{-1}$ in $40 \mathrm{~s}$ and a gradual decay to a red shift of $15 \mathrm{~km} \mathrm{~s}^{-1}$ in $100 \mathrm{~s}$, all accompanied by only $10 \%$ variations in the intensity of the emitting element.

During the third HRTS rocket flight, the slit was rastered across a 10" wide region of the quiet sun. The information in line profiles such as intensity, velocity and line width can then be assembled into images of these same quantities of a $10^{\mathrm{n}} \times 800^{\prime \prime}$ area of the Sun. These images show definite patterns or structures outlined by the intensity and velocity fields. Consequently, the C IV elements revealed by the time series are segments of these larger structures which are most likely organized by the magnetic field configuration. Images of the chromospheric line intensities coincide with dark $\mathrm{Ha}$ mottles while the C IV emission is produced at the tips of the mottles, roughly $2000 \mathrm{~km}$ above the chromospheric emission. Downflow patterns in $F$ e II often resemble similar downflow patterns in C IV, although there may be a spatial displacement of several thousands of $\mathrm{km}$ between the two.

\section{REFERENCES}

Boland, B.C., Engstrom, S.F.T., Jones, B.B. and Wilson, R. 1973, Astr. Astrophys., 22, 161.

Brueckner, G.E. and Bartoe, J.-D.F. 1983, Astrophys. J., 272, 329.

Dere, K.P., Bartoe, J.-D.F. and Brueckner, G.E. 1983, Astrophys. J. (Letters), 267, L65.

Dere, K.P., Bartoe, J.-D.F. and Brueckner, G.E. 1984, Astrophys. J., $281,870$.

Pneuman, G.W. and Kopp, R.A. 1977, Astr. Astrophys., 55, 305. 